\title{
Association Between the Admission Serum Bicarbonate and Short-Term and Long-Term Mortality in Acute Aortic Dissection Patients Admitted to the Intensive Care Unit
}

\author{
Liao Tan (iD) ${ }^{1,2}$ \\ Qian $X u^{3}$ \\ Chan $\mathrm{Li}^{2}$ \\ Xuliang Chen ${ }^{3}$ \\ Hui $\mathrm{Bai}^{3}$ \\ 'Department of Cardiology, The Third \\ Xiangya Hospital, Central South \\ University, Changsha, Hunan, People's \\ Republic of China; ${ }^{2}$ Department of \\ Cardiovascular Medicine, Xiangya \\ Hospital, Central South University, \\ Changsha, Hunan, People's Republic of \\ China; ${ }^{3}$ Department of Cardiovascular \\ Surgery, Xiangya Hospital, Central South \\ University, Changsha, Hunan, People's \\ Republic of China
}

\begin{abstract}
Objective: Serum bicarbonate $\left(\mathrm{HCO}^{-}\right)$level is strongly related to multiple cardiovascular complications. Currently, there is no study evaluating the prognostic ability of serum $\mathrm{HCO}^{-}$ level in intensive care unit (ICU) patients with acute aortic dissection (AAD). Hence, this study was to assess the relationship between admission serum $\mathrm{HCO}^{-}$level and clinical outcomes in patients with AAD.

Design, Settings and Participants: Clinical data were extracted from the MIMIC-III database. Cox proportional hazards models and Kaplan-Meier (KM) survival curve were used to evaluate the association between serum $\mathrm{HCO}^{-}$levels and short- and long-term mortality in ICU patients with AAD. The subgroup analysis and the receiver operating characteristic (ROC) curve analysis and further KM survival curve based on best cut-off value were applied to assessment of the performance of $\mathrm{HCO}^{-}$in predicting the mortality in each period ( 30 days, 90 days, 1 year and 5 years).
\end{abstract}

Main Results: Firstly, 336 eligible patients were trisected to low-HCO3 ${ }^{-}$level group $(<22 \mathrm{mmol} / \mathrm{L})$, mid-HCO3 ${ }^{-}$level group $(22-24 \mathrm{mmol} / \mathrm{L})$ and high-HCO3 ${ }^{-}$level group $(>24 \mathrm{mmol} / \mathrm{L})$. Then, in multivariate analysis, the serum $\mathrm{HCO}^{-}$of low levels $(<22 \mathrm{mmol} /$ L) was a significant risk predictor of all-cause mortality in 30 days, 90 days, 1 year and 5 years. Subgroup analyses indicated that there is no interaction in most strata. Finally, areas under ROC curve ranged from 0.60 to 0.69 .

Conclusion: The low $\mathrm{HCO}^{-}$serum level measured at ICU admission significantly predicts short-term and long-term mortality in AAD patients.

Keywords: serum bicarbonate, intensive care unit, acute aortic dissection, all-cause mortality

\section{Introduction}

Acute aortic dissection (AAD) is a devastating disease that needs emergent aortic replacement or repair surgery for survival. Due to its acute onset, rapid progression and high incidence of rupture of aorta, a majority of patients with AAD admitted to hospital had a loss of operation opportunity. ${ }^{1}$ Clinical data indicated that the mortality rate in patients with AAD within 48 hours of admission to the intensive care unit (ICU) ranged from $36 \%$ to $72 \%$, with an increase of $1-2 \%$ per hour. ${ }^{2}$ Hence, identification of simple biomarkers for evaluating the prognosis of AAD plays a vital role in risk stratification in this kind of patient. Previous studies have
Correspondence: Hui Bai

Department of Cardiovascular Surgery, Xiangya Hospital, Central South

University, 87 Xiangya Road, Changsha,

410013, People's Republic of China

Email22II200403@qq.com 
shown that lymphocyte-to-monocyte ratio (LMR), ${ }^{3}$ hypotensive systolic blood pressure, ${ }^{4} \mathrm{~N}$-terminal pro-brain natriuretic peptide, ${ }^{5}$ C-reactive protein, ${ }^{6}$ cardiac troponin ${ }^{7}$ and serum fibrinogen level ${ }^{8}$ were associated with the prognosis of patients with AAD; however, the studies above mainly used in-hospital mortality as outcomes. Effective variables to predict the prognosis of AAD are still lacking in clinical practice. Thus, more new risk factors should be explored and longer follow-up time to be validated in future study of AAD.

Serum bicarbonate $\left(\mathrm{HCO}^{-}\right)$is a biomarker of the homeostasis of acid-base. Abnormal serum $\mathrm{HCO}^{-}$is mainly caused by respiratory disorder or kidney dysfunction. Previous studies mainly focused on the relationship between mortality of renal disease and serum $\mathrm{HCO}^{-}$ level. ${ }^{9}$ Recently, serum $\mathrm{HCO}^{-}$level was reported to be strongly related to multiple complications and mortality of cardiovascular diseases like stroke and myocardial infarction. ${ }^{10}$ In a large cohort of US adults, the hazard for cardiovascular mortality was increased by $8 \%$ for every $1 \mathrm{mEq} / \mathrm{L}$ increase in serum $\mathrm{HCO}^{-}$level above 26 $\mathrm{mEq} / \mathrm{L} .^{11}$ However, the relationship between serum $\mathrm{HCO}^{-}$level and clinical outcomes in AAD patients is still unknown. This study was instigated to assess the association between serum $\mathrm{HCO}^{-}$levels on admission and short-term and long-term mortality in AAD patients in ICU.

\section{Methods}

\section{Database Source}

We acquired clinical data from the MIMIC-III database which contains comprehensive information on ICU admissions for over 60,000 patients from 2001 to $2012 .^{12}$ The data include general information, treatment process and survival data. This study on human participants obtained the approval of the Massachusetts Institute of Technology (MIT) ethics committee. Author Tan completed the National Institutes of Health web-based training course "Protecting Human Research Participants" and gained access to the database (Certification Number: 35950815).

\section{Study Population Criteria}

According to the ninth revision of the International Classification of Diseases (ICD9) adopted in the MIMIC III database, we selected adult patients with Stanford A and B types of AAD. Patients were excluded as follows: 1) patients with multiple admission records; 2) no serum $\mathrm{HCO}^{-}$data; 3) missing more than 5\% individual data. Complete selection process is indicated in Figure 1.

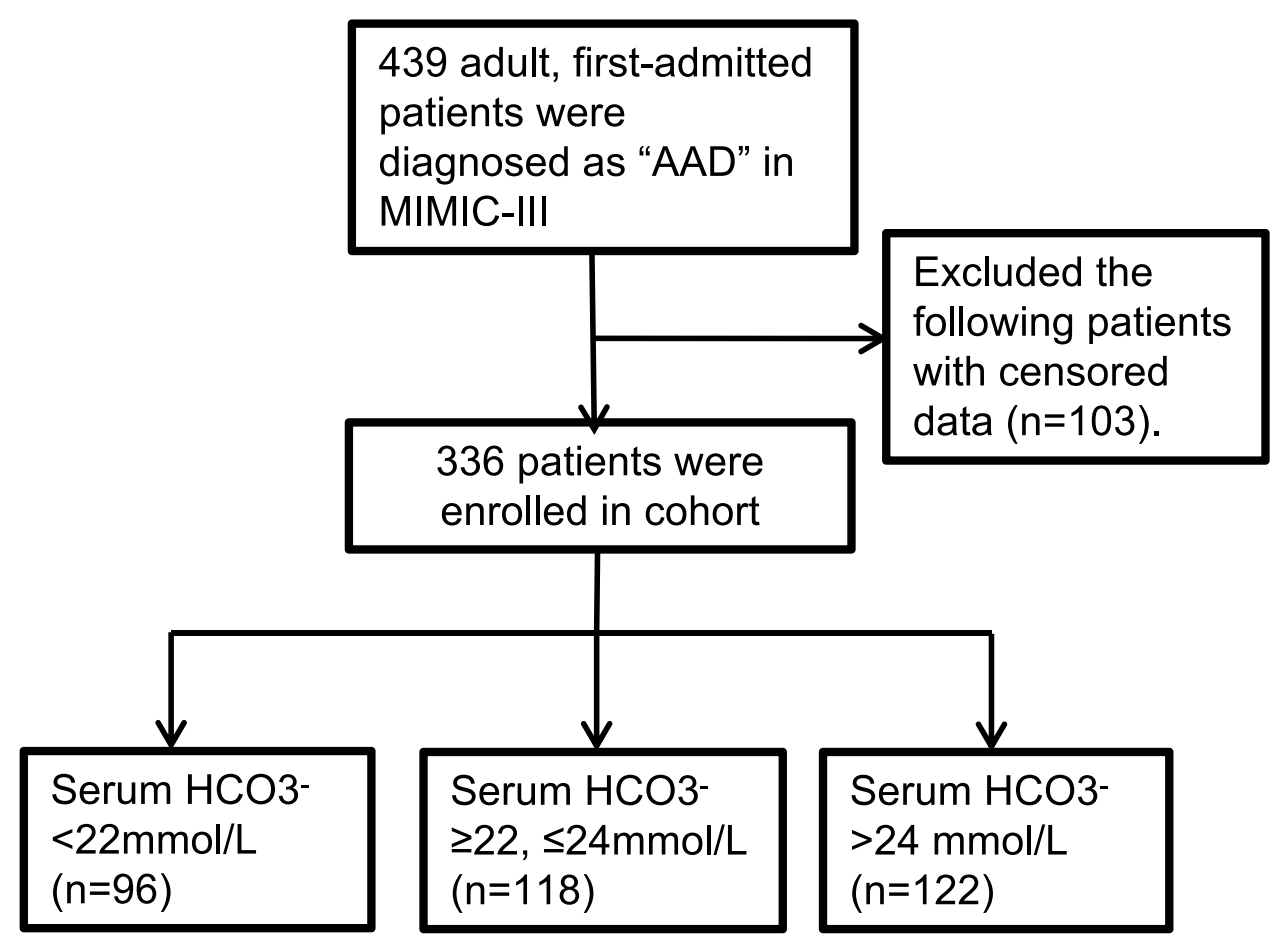

Figure I The flow chart describing the procedure used for patients selection. 
Table I Characteristics of Subjects

\begin{tabular}{|c|c|c|c|c|c|}
\hline \multirow[t]{2}{*}{ Characteristics } & \multirow[t]{2}{*}{ Total $(n=336)$} & \multicolumn{4}{|c|}{ Serum $\mathrm{HCO}^{-}$Levels $(\mathrm{mmol} / \mathrm{L})$} \\
\hline & & $<22(n=96)$ & $22-24(n=118)$ & $>24(n=122)$ & $P$ value \\
\hline Gender & & & & & 0.764 \\
\hline Male & $213(63.39 \%)$ & $61(63.54 \%)$ & $72(61.02 \%)$ & $80(65.57 \%)$ & \\
\hline Female & $123(36.61 \%)$ & 35 (36.46\%) & $46(38.98 \%)$ & $42(34.43 \%)$ & \\
\hline Age, years & $69.00(56.00-78.00)$ & $67.00(54.75-78.00)$ & $70.50(59.00-80.00)$ & $71.00(57.25-77.00)$ & 0.347 \\
\hline Insurance & & & & & 0.55 \\
\hline Government & 20 (5.95\%) & $5(5.21 \%)$ & 7 (5.93\%) & $8(6.56 \%)$ & \\
\hline Medicaid & 17 (5.06\%) & 7 (7.29\%) & $3(2.54 \%)$ & 7 (5.74\%) & \\
\hline Medicare & $176(52.38 \%)$ & 46 (47.92\%) & $63(53.39 \%)$ & 67 (54.92\%) & \\
\hline Private & 118 (35.12\%) & $38(39.58 \%)$ & $43(36.44 \%)$ & 37 (30.33\%) & \\
\hline Selfpay & $5(1.49 \%)$ & $0(0.00 \%)$ & $2(1.69 \%)$ & $3(2.46 \%)$ & \\
\hline Ethnicity & & & & & 0.261 \\
\hline White & $233(69.35 \%)$ & 69 (7I.88\%) & 88 (74.58\%) & $76(62.30 \%)$ & \\
\hline Black & $44(13.19 \%)$ & II (II.46\%) & 15 (12.71\%) & I8 (I4.75\%) & \\
\hline Hispanic & $13(3.87 \%)$ & 5 (5.2।\%) & 4 (3.39\%) & $4(3.28 \%)$ & \\
\hline Others & 46 (13.69\%) & II (II.46\%) & II (9.32\%) & 24 (19.67\%) & \\
\hline Stanford type & & & & & 0.003 \\
\hline A type & 230 (68.45\%) & 68 (70.83\%) & 75 (63.56\%) & 87 (7I.3।\%) & \\
\hline B type & $106(31,55 \%)$ & $28(29.17 \%)$ & 43 (36.44\%) & 35 (28.69\%) & \\
\hline Dissection site & & & & & 0.392 \\
\hline Thoracic & I 87 (55.65\%) & $56(58.33 \%)$ & 68 (57.63\%) & $63(51.64 \%)$ & \\
\hline Abdominal & 62 (18.45\%) & $2 \mathrm{I}(2 \mathrm{I}, 88 \%)$ & 21 (17.80\%) & $21(17.21 \%)$ & \\
\hline Thoracoabdominal & 87 (25.89\%) & 19 (19.79\%) & 30 (25.42\%) & 38 (3I. $15 \%)$ & \\
\hline Aorta replacement & 198 (58.93\%) & 57 (59.38\%) & 72 (6I.02\%) & 69 (56.56\%) & 0.777 \\
\hline ICU MV & 170 (50.60\%) & 69 (7I.88\%) & 60 (50.85\%) & 79 (64.75\%) & 0.005 \\
\hline Comorbidities & & & & & \\
\hline Hypertension & 44 (13.1\%) & 46 (47.92\%) & $80(67.80 \%)$ & 73 (59.83\%) & 0.013 \\
\hline Diabetes & $30(8.93 \%)$ & II (II.46\%) & $10(8.47 \%)$ & 9 (7.38\%) & 0.564 \\
\hline $\mathrm{HC}$ & 53 (I5.77\%) & $16(16.67 \%)$ & $20(16.95 \%)$ & 17 (I3.93\%) & 0.782 \\
\hline Valvular disease & 33 (9.82\%) & 9 (9.38\%) & II (9.32\%) & $13(10.66 \%)$ & 0.927 \\
\hline Maligancy & II (3.27\%) & $3(3.13 \%)$ & $4(3.39 \%)$ & $4(3.28 \%)$ & 0.994 \\
\hline Stroke & 26 (7.74\%) & $9(9.38 \%)$ & $8(6.78 \%)$ & $9(7.38 \%)$ & 0.765 \\
\hline CAD & $60(17.86 \%)$ & 25 (26.04\%) & 17 (14.4I\%) & 18 (14.75\%) & 0.046 \\
\hline $\mathrm{CHF}$ & 31 (9.23\%) & $10(10.42 \%)$ & II (9.32\%) & $10(8.20 \%)$ & 0.853 \\
\hline Atrial fibrillation & 57 (16.96\%) & $16(16.67 \%)$ & $20(16.95 \%)$ & 21 (36.84\%) & 0.994 \\
\hline Renal disease & 45 (13.39\%) & $15(15.63 \%)$ & 15 (12.71\%) & $15(12.30 \%)$ & 0.745 \\
\hline Liver disease & $10(2.98 \%)$ & $2(2.08 \%)$ & $4(3.39 \%)$ & $4(3.28 \%)$ & 0.83 \\
\hline Respiratory disease & 64 (19.05\%) & $12(12.50 \%)$ & 17 (14.4I\%) & 35 (28.69\%) & 0.003 \\
\hline $\mathrm{AKI}$ & 135 (40.18\%) & $50(52.08 \%)$ & $43(36.44 \%)$ & $42(34.43 \%)$ & 0.018 \\
\hline RRT & $29(8.63 \%)$ & $13(13.54 \%)$ & $8(6.78 \%)$ & $8(6.56 \%)$ & 0.127 \\
\hline Laboratory variables & & & & & \\
\hline WBC, $10^{3} / \mu \mathrm{L}$ & $8.80(6.20-11.00)$ & $9.05(5.90-12.43)$ & $9.00(6.85-10.80)$ & $8.45(6.05-10.38)$ & 0.192 \\
\hline $\mathrm{RBC}, 10^{3} / \mu \mathrm{L}$ & $3.66(2.26-4.16)$ & $3.52(3.22-3.92)$ & $3.64(3.29-4.11)$ & $3.82(3.29-4.33)$ & 0.03 \\
\hline PLT, $10^{3} / \mu \mathrm{L}$ & $164(119-228)$ & $139(93.75-193.50)$ & 159 (123-224.75) & 185.5 (I 28.5-273.75) & $<0.001$ \\
\hline RDW, \% & $14.40(13.60-15.30)$ & $14.50(13.60-15.30)$ & $14.35(13.50-15.30)$ & $14.40(\mid 3.73-15.50)$ & 0.85 \\
\hline Hematocrit, \% & $28.95(23.00-33.80)$ & $26.65(21.98-30.78)$ & $28.75(23.75-33.95)$ & $30.55(23.68-34.20)$ & 0.02 \\
\hline $\mathrm{Hb}, \mathrm{g} / \mathrm{dL}$ & $9.75(7.80-11.60)$ & 9.10 (7.38-10.53) & 9.85 (7.93-11.60) & $10.25(8.15-11.70)$ & 0.023 \\
\hline
\end{tabular}

(Continued) 
Table I (Continued).

\begin{tabular}{|c|c|c|c|c|c|}
\hline \multirow[t]{2}{*}{ Characteristics } & \multirow[t]{2}{*}{ Total $(n=336)$} & \multicolumn{4}{|c|}{ Serum $\mathrm{HCO}^{-}$Levels $(\mathrm{mmol} / \mathrm{L})$} \\
\hline & & $<22(n=96)$ & $22-24(n=118)$ & $>24(n=122)$ & $P$ value \\
\hline Anion gap, $\mathrm{mmol} / \mathrm{L}$ & $12.50(10.00-14.00)$ & $14.00(11.00-16.00)$ & $13.00(10.00-14.00)$ & $12.00(10.00-13.00)$ & $<0.001$ \\
\hline Lactate, $\mathrm{mmol} / \mathrm{L}$ & $3.20(1.70-5.85)$ & $4.10(2.20-6.88)$ & $3.60(1.70-5.80)$ & $2.00(1.30-3.75)$ & $<0.001$ \\
\hline $\mathrm{pH}$ value & $7.39(7.33-7.43)$ & $7.37(7.27-7.42)$ & 7.39 (7.34-7.44) & 7.41 (7.36-7.44) & $<0.001$ \\
\hline $\mathrm{BUN}, \mathrm{mg} / \mathrm{dL}$ & $16.00(12.00-22.00)$ & $18.00(13.00-26.00)$ & $16.50(12.00-20.75)$ & $16.00(11.00-20.75)$ & 0.037 \\
\hline Creatinine, $\mathrm{mg} / \mathrm{dL}$ & $1.05(0.80-1.53)$ & $1.20(0.90-1.90)$ & $\mathrm{I} .00(0.80-\mathrm{I} .40)$ & $0.95(0.73-1.30)$ & $<0.001$ \\
\hline Chloride, $\mathrm{mmol} / \mathrm{L}$ & $103(100-105)$ & $104(101.75-106)$ & $103(101-105)$ & I0I (99-104) & $<0.001$ \\
\hline Glucose, mg/dL & $103(89.00-119.25)$ & $101(86-119)$ & $104(88-118)$ & $102(93-121.75)$ & 0.541 \\
\hline APTT, s & $28.15(25.20-32.63)$ & $28.70(25.50-36.20)$ & $27.35(24.50-31.38)$ & $28.75(25.50-32.00)$ & 0.47 \\
\hline PT, s & $13.40(12.50-14.40)$ & $13.50(12.40-14.53)$ & $13.35(12.70-14.10)$ & $13.30(12.50-14.60)$ & 0.932 \\
\hline INR & $1.20(1.10-1.30)$ & $1.20(1.10-1.30)$ & $1.20(1.10-1.30)$ & $1.20(1.10-1.30)$ & 0.836 \\
\hline Sodium, $\mathrm{mmol} / \mathrm{L}$ & $137(134-139)$ & $135.50(133-138)$ & $136(134-139)$ & 137.50 (135-139) & 0.001 \\
\hline Potassium, $\mathrm{mmol} / \mathrm{L}$ & $3.60(3.30-3.90)$ & $3.60(3.30-3.90)$ & $3.70(3.40-4.00)$ & $3.60(3.30-3.90)$ & 0.264 \\
\hline Total Ca, mg/dL & $8.50(8.10-9.00)$ & $8.50(7.90-8.83)$ & $8.50(8.10-9.00)$ & $8.60(8.30-9.10)$ & 0.02 \\
\hline Magnesium, mg/dL & $2.00(1.90-2.30)$ & $2.10(1.90-2.40)$ & $2.00(1.90-2.30)$ & $2.00(1.90-2.20)$ & 0.439 \\
\hline \multicolumn{6}{|l|}{ Vital signs } \\
\hline $\mathrm{SBP}, \mathrm{mmHg}$ & $151(136-165)$ & $149.50(134-168)$ & I5I (136-I67.50) & $150.50(136-162)$ & 0.526 \\
\hline $\mathrm{DBP}, \mathrm{mmHg}$ & 81.00 (72 0.00-91.00) & $81.00(72.00-94.00)$ & $81.00(72.00-94.00)$ & $85.50(72.25-87.00)$ & 0.604 \\
\hline $\mathrm{MBP}, \mathrm{mmHg}$ & $102(93-113)$ & $101.50(95-114.25)$ & $103(92.25-117.50)$ & I0I (9I.75-I09.75) & 0.193 \\
\hline $\mathrm{HR}$, beats/min & $90(80-102.25)$ & $95.00(84.00-1 \mid 12.00)$ & 90.50 (79.25-99.75) & $87.50(78.00-101.00)$ & 0.015 \\
\hline $\mathrm{RR}$, times/min & $25.00(22.00-29.00)$ & $26.00(23.00-30.00)$ & $24.00(22.00-29.00)$ & $26.00(22.00-29.00)$ & 0.201 \\
\hline Temperature, ${ }^{\circ} \mathrm{C}$ & $37.28(36.78-37.83)$ & $37.30(36.72-38.01)$ & 37.31 (36.78-37.89) & $37.22(36.89-37.70)$ & 0.942 \\
\hline $\mathrm{SPO}_{2}, \%$ & $92.00(90.00-94.00)$ & $92.00(01.00-94.00)$ & $92.00(91.00-94.00)$ & $92.00(90.00-94.00)$ & 0.365 \\
\hline \multicolumn{6}{|l|}{ Severity score } \\
\hline SAPSII & $35.00(28.00-43.00)$ & $43.00(33.75-53.50)$ & $34.00(28.25-39.00)$ & $31.00(25.00-39.00)$ & $<0.001$ \\
\hline OASIS & $32.00(26.75-39.00)$ & $37.00(32.00-42.25)$ & $31.00(26.00-37.00)$ & $29.50(25.00-34.00)$ & $<0.001$ \\
\hline SOFA & $4.00(2.00-6.00)$ & $6.00(3.75-9.00)$ & $3.00(2.00-5.00)$ & $3.00(1.00-5.00)$ & $<0.001$ \\
\hline SIRS & $3.00(2.00-3.00)$ & $3.00(2.00-4.00)$ & $3.00(2.00-3.00)$ & $2.00(1.00-3.00)$ & $<0.001$ \\
\hline
\end{tabular}

Abbreviations: ICU MV, intensive care unit mechanical ventilation; HC, hypercholesterolemia; CAD, coronary artery disease; CHF, congestive heart failure; AKI, acute kidney injury; RRT, renal replacement therapy; WBC, white blood cell; RBC, red blood cell; PLT, platelet; RDW, red cell distribution width; BUN, blood urea nitrogen; APTT, activated partial thromboplastin time; PT, prothrombin time; INR, international normalized ratio; Total Ca, total calcium; SBP, systolic blood pressure; DBP, diastolic blood pressure; MBP, mean blood pressure; HR, heart rate; RR, respiratory rate; SAPS II, simplified acute physiology score; OASIS, Oxford acute severity of illness score; SOFA, sequential organ failure assessment; SIRS, systemic inflammatory response syndrome.

\section{Data Stratification}

Similar to previous studies, we used Structured Query Language (SQL) with PostgreSQL 9.6 to extract data. Since some patients had multiple results of $\mathrm{HCO}^{-}$measurement, we selected their first result after ICU admission. Other data, including demographics, vital signs and laboratory tests, were also extracted. Vital signs included heart rate (HR), respiratory rate (RR), temperature, $\mathrm{SPO}_{2}$, systolic blood pressure (SBP), diastolic blood pressure (DBP) and mean blood pressure (MBP); the laboratory variables included venous serum $\mathrm{HCO}^{-}$, lactate, anion gap, $\mathrm{pH}$ value, etc. Comorbidities were also collected, including hypertension, diabetes, malignancy, renal disease, etc. Severity of illness was captured by severity scores such as simplified acute physiology score (SAPSII), Oxford acute severity of illness score (OASIS), sequential organ failure assessment (SOFA) and systemic inflammatory response syndrome (SIRS) score. Others included age, gender, Stanford type, dissection site, surgery of aorta replacement, ICU mechanical ventilation (MV) and survival time.

\section{Statistical Analysis}

Baseline characteristics of AAD patients were stratified to tertiles including low- $\mathrm{HCO}^{-}$, mid- $\mathrm{HCO}^{-}$and high$\mathrm{HCO}^{-}$. Normally distributed continuous variables were 


\section{KM survival curve}

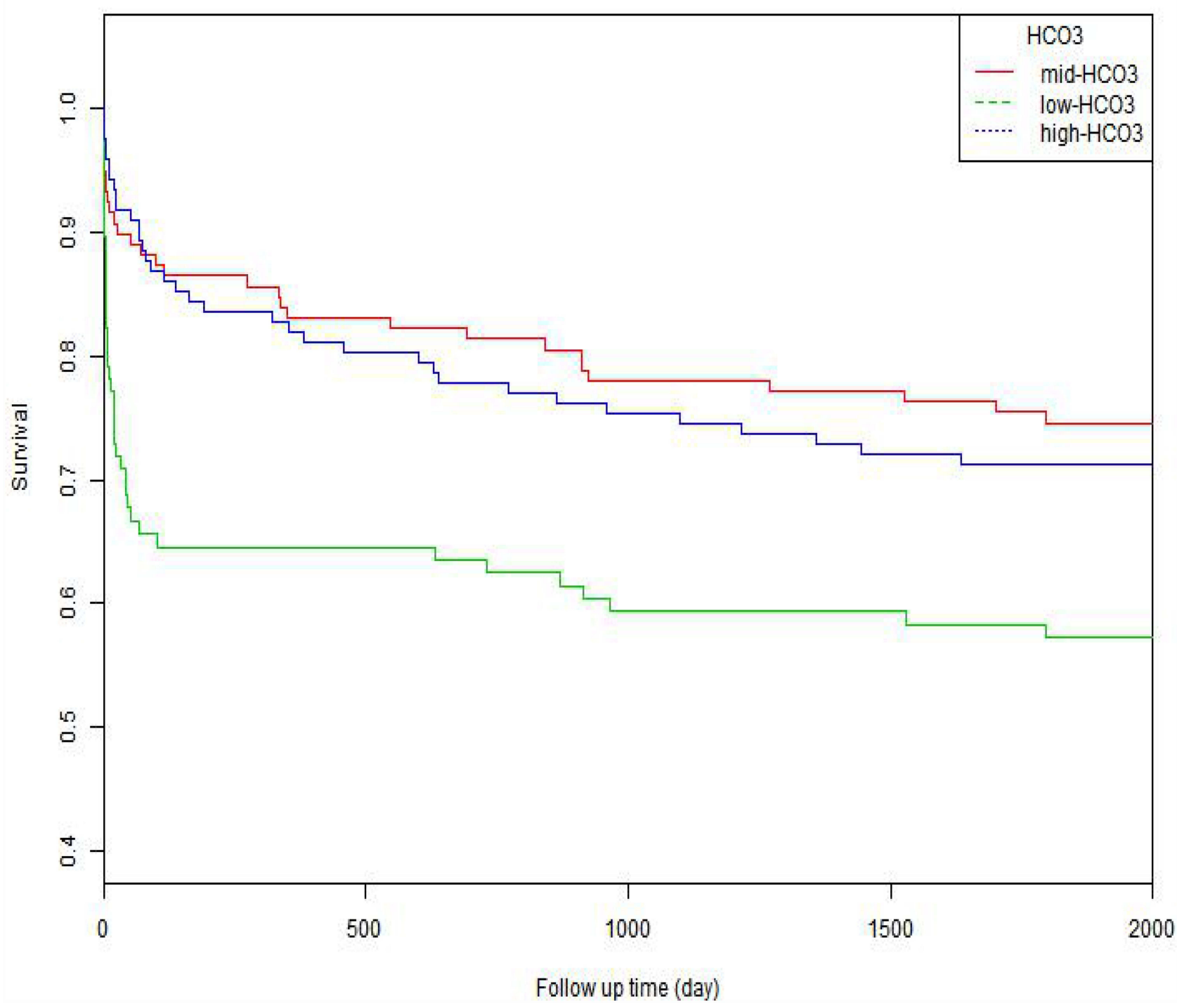

Figure 2 The $\mathrm{KM}$ survival curve of low- $\mathrm{HCO}^{-}$, mid- $\mathrm{HCO}^{-}$and high- $\mathrm{HCO}^{-}$groups.

presented as the means $\pm \mathrm{SD}$, while other continuous variables were reported as the median and interquartile range (IQR). Categorical data were presented as total number and percentages, which were compared by the chi-square test to determine differences among the tertiles. Then, covariate selection and multi-collinearity check analysis were conducted. Multifactorial Cox regression models were performed to evaluate the predictive value of serum $\mathrm{HCO}^{-}$in each period's mortality (30 days, 90 days, 1 year and 5 years) with hazard ratios (HRs) and 95\% confidence intervals (CIs). In multivariate analysis, we used 2 adjusted multivariate models, and covariates were adjusted to age, gender, ethnicity, insurance, Stanford type, dissection site, surgery of aorta replacement, ICU MV, etc. Besides, we used the log rank tests to compare the survival rates in low- $\mathrm{HCO}^{-}$, mid- $\mathrm{HCO}^{-}$and high- $\mathrm{HCO}^{-}$groups and report the results with Kaplan-Meier curves. Subgroup analyses were conducted to evaluate the relationship between the $\mathrm{HCO}^{-}$level and 30-day mortality among the different subgroups, including age, gender, ethnicity, insurance, Stanford type, dissection site, surgery of aorta replacement, ICU MV, etc. Finally, we used ROC curve and calculated the value of AUC and best cut-off value according to the Youden index to test the effectiveness of $\mathrm{HCO}^{-}$in predicting short-term and long-term mortality. The KM survival curve based on the best cut- 
Table 2 HRs (95\% Cls) for Mortality Across Groups of Serum HCO3

\begin{tabular}{|c|c|c|c|c|c|c|}
\hline \multirow[t]{2}{*}{ Serum $\mathrm{HCO}^{-}$Level } & \multicolumn{2}{|c|}{ Non-Adjusted } & \multicolumn{2}{|c|}{ Model I } & \multicolumn{2}{|c|}{ Model 2} \\
\hline & HR (95\% Cls) & $P$ value & HR (95\% Cls) & $P$ value & HR (95\% Cls) & $P$ value \\
\hline \multicolumn{7}{|l|}{ 30-day mortality } \\
\hline \multicolumn{7}{|l|}{$\mathrm{HCO}^{-}, \mathrm{mmol} / \mathrm{L}$} \\
\hline$<22 \mathrm{mmol} / \mathrm{L}$ & $3.54(1.67,7.49)$ & $<0.001$ & $3.54(1.67,7.49)$ & 0.001 & $5.1 I(I .4 I, I 8.46)$ & 0.013 \\
\hline $22-24 \mathrm{mmol} / \mathrm{L}$ & 1.0 (ref) & 1 & 1.0 (ref) & I & 1.0 (ref) & 1 \\
\hline$>24 \mathrm{mmol} / \mathrm{L}$ & $0.77(0.32,1.87)$ & 0.567 & $0.74(0.30,1.82)$ & 0.507 & $0.75(0.20,2.78)$ & 0.663 \\
\hline \multicolumn{7}{|l|}{ 90-day mortality } \\
\hline \multicolumn{7}{|l|}{$\mathrm{HCO}^{-}, \mathrm{mmol} / \mathrm{L}$} \\
\hline$<22 \mathrm{mmol} / \mathrm{L}$ & $3.95(1.96,7.98)$ & $<0.001$ & $3.95(1.93,8.08)$ & $<0.001$ & $5.58(1.86,16.77)$ & 0.002 \\
\hline $22-24 \mathrm{mmol} / \mathrm{L}$ & 1.0 (ref) & I & I.0 (ref) & I & $1.0(\mathrm{ref})$ & 1 \\
\hline$>24 \mathrm{mmol} / \mathrm{L}$ & $1.02(0.47,2.23)$ & 0.951 & $0.98(0.44,2.19)$ & 0.969 & $1.49(0.49,4.54)$ & 0.486 \\
\hline \multicolumn{7}{|l|}{ I-year mortality } \\
\hline \multicolumn{7}{|l|}{$\mathrm{HCO}^{-}, \mathrm{mmol} / \mathrm{L}$} \\
\hline$<22 \mathrm{mmol} / \mathrm{L}$ & $2.71(1.43,5.13)$ & 0.002 & $2.67(1.39,5.13)$ & 0.003 & $3.30(1.24,8.78)$ & 0.016 \\
\hline $22-24 \mathrm{mmol} / \mathrm{L}$ & 1.0 (ref) & I & $\mathrm{I} .0(\mathrm{ref})$ & I & $\mathrm{I} .0$ (ref) & I \\
\hline$>24 \mathrm{mmol} / \mathrm{L}$ & $1.06(0.54,2.07)$ & 0.864 & $1.06(0.53,2.10)$ & 0.877 & I.5I $(0.57,3.98)$ & 0.402 \\
\hline \multicolumn{7}{|l|}{ 5-year mortality } \\
\hline \multicolumn{7}{|l|}{$\mathrm{HCO}^{-}, \mathrm{mmol} / \mathrm{L}$} \\
\hline$<22 \mathrm{mmol} / \mathrm{L}$ & $2.19(1.23,3.91)$ & 0.008 & $2.13(1.18,3.83)$ & 0.012 & $4.08(1.70,9.78)$ & 0.002 \\
\hline $22-24 \mathrm{mmol} / \mathrm{L}$ & 1.0 (ref) & I & 1.0 (ref) & 1 & 1.0 (ref) & 1 \\
\hline$>24 \mathrm{mmol} / \mathrm{L}$ & I.18 $(0.67,2.09)$ & 0.574 & $\mathrm{I} .14(0.63,2.04)$ & 0.668 & I.67 $(0.75,3.72)$ & 0.209 \\
\hline
\end{tabular}

Notes: Models were derived from Cox proportional hazards regression models. Non-adjusted model adjusted for: none. Model I adjusted for: gender; age; ethnicity and insurance. Model 2 adjusted for: age; gender; ethnicity; insurance; Stanford type; dissection site; ICU MV; therapy type; hypertension; diabetes; hypercholesterolemia; valvular disease; malignancy; stroke; CAD; CHF; atrial fibrillation; renal disease; liver disease; respiratory disease; AKI; WBC; RDW; RBC; Hb; PLT; BUN; creatinine; chloride; glucose; APTT; INR; sodium; potassium; total Ca; magnesium; anion gap; PH value; SBP; DBP; MBP; HR; RR; temperature and SPO 2 .

off value was generated. Finally, the KM survival curve based on the best cut-off value was generated, and the comparison within $\mathrm{HCO}^{-}$, anion gap and lactate was conducted. All statistical analyses were conducted using the EmpowerStats ver 2.17.8 and R software version 3.42, and $P$ value $<0.05$ was considered statistically significant.

\section{Results}

\section{Characteristics of the Subjects}

Overall, 336 eligible AAD patients were included in the study. Figure 1 is a flow chart describing the procedure of patient selection. Table 1 lists the baseline characteristics of the patients. Based on the value of serum $\mathrm{HCO}^{-}$, patients were categorized in trisected groups including low-HCO3 ${ }^{-}$group, mid-HCO3 ${ }^{-}$group and high-HCO3 ${ }^{-}$ group ( $<22 \mathrm{mmol} / \mathrm{L} ; 22-24 \mathrm{mmol} / \mathrm{L} ;>24 \mathrm{mmol} / \mathrm{L})$. Patients with a high serum $\mathrm{HCO}^{-}$level were more likely to have lower platelet (PLT), hematocrit, hemoglobin $(\mathrm{Hb})$, creatinine, sodium, chloride; and higher red blood cell $(\mathrm{RBC})$, lactate, anion gap, $\mathrm{pH}$ value, blood urea nitrogen (BUN), total calcium. In addition, patients with low $\mathrm{HCO}^{-}$level had less hypertension and higher severity scores.

\section{Low-HCO3 ${ }^{-}$Is Directly Related to the Short-Term and Long-Term Mortality of AAD}

During the 5 years of follow-up, 106 (31.55\%) patients died. The number of all-cause fatalities in the low $(n=96)$, mid $(n=118)$ and high $(n=122) \mathrm{HCO}^{-}$groups were 41 (42.71\%), $30(25.42 \%)$ and 35 (28.69\%), respectively. Figure 2 shows the Kaplan-Meier curve for patients in the low, mid and high $\mathrm{HCO}^{-}$groups. The survival rate of the low- $\mathrm{HCO}^{-}$group is lower than the other two groups.

To explore the relationship between serum $\mathrm{HCO}^{-}$ level and short-term and long-term mortality, multivariate Cox regression analysis was conducted and listed in Table 2. First of all, according to the results of covariates selection and multi-collinearity check analysis, 
Table 3 Subgroup Analysis of the Associations Between Serum $\mathrm{HCO}^{-}$and 30-Day All-Cause Mortality

\begin{tabular}{|c|c|c|c|c|c|}
\hline & \multirow[t]{2}{*}{ No. of Patients } & \multicolumn{3}{|c|}{ Serum $\mathrm{HCO}^{-}$Levels $(\mathrm{mmol} / \mathrm{L})$} & \multirow[t]{2}{*}{$P$ for Interaction } \\
\hline & & $<22$ & $22-24$ & $>24$ & \\
\hline Gender & & & & & 0.828 \\
\hline Male & 213 & $2.96(1.29,6.80)$ & ref & $0.66(0.23,1.89)$ & \\
\hline Female & 123 & $3.25(1.00,10.56)$ & ref & $1.09(0.27,4.38)$ & \\
\hline Age, years & & & & & 0.924 \\
\hline$<69$ & 165 & $3.18(1.26,8.01)$ & ref & $0.92(0.30,2.85)$ & \\
\hline$\geq 69$ & 171 & $2.66(0.95,7.48)$ & ref & $0.65 \mathrm{I}(0.18,2.3 \mathrm{I})$ & \\
\hline Insurance & & & & & l \\
\hline Government & 20 & 1 & ref & 1 & \\
\hline Medicaid & 17 & 1 & ref & 1 & \\
\hline Medicare & 176 & $2.32(0.90,5.99)$ & ref & $0.39(0.10,1.53)$ & \\
\hline Private & 118 & $3.66(1.32,10.16)$ & ref & $0.91(0.24,3.37)$ & \\
\hline Selfpay & 5 & 1 & ref & $1.00(1.00,1.00)$ & \\
\hline Ethnicity & & & & & 0.89 \\
\hline White & 233 & $3.18(1.38,7.33)$ & ref & $0.87(0.30,2.49)$ & \\
\hline Black & 44 & $1.38(0.28,6.84)$ & ref & $0.49(0.08,2.94)$ & \\
\hline Hispanic & 13 & 1 & ref & 1 & \\
\hline Others & 46 & $5.88(0.69,50.40)$ & ref & $0.91(0.08,10.01)$ & \\
\hline Stanford type & & & & & 0.387 \\
\hline A type & 230 & $8.50(1.05,16.26)$ & ref & $0.95(0.06,16.29)$ & \\
\hline B type & 106 & $2.27(1.17,5.94)$ & ref & $0.65(0.20,2.09)$ & \\
\hline Aorta replacement & & & & & 0.768 \\
\hline No & 138 & $5.27(1.44,8.94)$ & ref & $0.64(0.72,3.16)$ & \\
\hline Yes & 198 & $2.75(0.98,7.13)$ & ref & $0.54(0.17,2.83)$ & \\
\hline ICU MV & & & & & 0.753 \\
\hline No & 166 & $3.57(2.26,5.23)$ & ref & $0.79(0.33,2.54)$ & \\
\hline Yes & 170 & $4.92(1.67,9.91)$ & ref & $0.88(0.12,4.58)$ & \\
\hline Hypertension & & & & & 0.179 \\
\hline No & 137 & $1.77(0.72,4.35)$ & ref & $0.32(0.08,1.22)$ & \\
\hline Yes & 199 & $4.64(1.63,13.17)$ & ref & $\mathrm{I} .53(0.48,4.8 \mathrm{I})$ & \\
\hline Diabetes & & & & & 0.091 \\
\hline No & 306 & $2.572(1.27,5.20)$ & ref & $0.78(0.34,1.8 \mathrm{I})$ & \\
\hline Yes & 30 & 1 & ref & 1 & \\
\hline Hypercholesterolemia & & & & & 0.156 \\
\hline No & 283 & $3.56(1.64,7.69)$ & ref & $1.04(0.42,2.55)$ & \\
\hline Yes & 53 & $1.597(0.36,7.14)$ & ref & 1 & \\
\hline Valvular disease & & & & & 0.668 \\
\hline No & 303 & $3.00(1.42,6.33)$ & ref & $0.87(0.36,2.15)$ & \\
\hline Yes & 33 & $3.36(0.65,17.39)$ & ref & $0.38(0.03,4.19)$ & \\
\hline Malignancy & & & & & 0.478 \\
\hline No & 325 & $2.93(\mathrm{I} .48,5.8 \mathrm{I})$ & ref & $0.79(0.34,1.83)$ & \\
\hline Yes & 11 & 1 & ref & 1 & \\
\hline Stroke & & & & & 0.891 \\
\hline No & 310 & $3.22(1.47,7.02)$ & ref & $0.85(0.33,2.21)$ & \\
\hline
\end{tabular}

(Continued) 
Table 3 (Continued).

\begin{tabular}{|c|c|c|c|c|c|}
\hline & \multirow[t]{2}{*}{ No. of Patients } & \multicolumn{3}{|c|}{ Serum $\mathrm{HCO}^{-}$Levels $(\mathrm{mmol} / \mathrm{L})$} & \multirow[t]{2}{*}{$P$ for Interaction } \\
\hline & & $<22$ & $22-24$ & $>24$ & \\
\hline Yes & 26 & $2.27(0.56,9.18)$ & ref & $0.54(0.09,3.23)$ & \\
\hline CAD & & & & & 0.16 \\
\hline No & 276 & $4.25(1.88,9.59)$ & ref & $1.09(0.42,2.8 \mathrm{I})$ & \\
\hline Yes & 60 & $1.00(0.28,3.54)$ & ref & $0.21(0.02,1.90)$ & \\
\hline $\mathrm{CHF}$ & & & & & 0.307 \\
\hline No & 305 & $3.29(1.57,6.88)$ & ref & $0.94(0.39,2.25)$ & \\
\hline Yes & 31 & $1.91(0.32,11.43)$ & ref & 1 & \\
\hline Atrial fibrillation & & & & & 0.735 \\
\hline No & 279 & $3.49(1.62,7.55)$ & ref & $0.84(0.32,2.18)$ & \\
\hline Yes & 57 & $1.80(0.40,8.03)$ & ref & $0.64(0.11,3.83)$ & \\
\hline Renal disease & & & & & 0.094 \\
\hline No & 291 & $3.16(1.44,6.94)$ & ref & $1.07(0.43,2.62)$ & \\
\hline Yes & 45 & $2.35(0.61,9.13)$ & ref & 1 & \\
\hline Liver disease & & & & & 0.049 \\
\hline No & 326 & $2.77(I .39,5.5 I)$ & ref & $0.79(0.34, I .83)$ & \\
\hline Yes & 10 & 1 & ref & 1 & \\
\hline Respiratory disease & & & & & 0.727 \\
\hline No & 272 & $3.51(1.55,7.92)$ & ref & $0.87(0.30,2.50)$ & \\
\hline Yes & 64 & $2.21(0.62,7.86)$ & ref & $0.42(0.11,1.69)$ & \\
\hline AKI & & & & & 0.507 \\
\hline No & 201 & $3.10(1.22,7.87)$ & ref & $1.06(0.39,2.93)$ & \\
\hline Yes & 135 & $2.83(1.03,7.80)$ & ref & $0.40(0.08,2.03)$ & \\
\hline RRT & & & & & 0.418 \\
\hline No & 307 & $2.94(1.43,6.06)$ & ref & $0.86(0.37,2.03)$ & \\
\hline Yes & 29 & $3.31(0.39,28.38)$ & ref & 1 & \\
\hline WBC, $10^{3} / \mu \mathrm{L}$ & & & & & 0.84 \\
\hline$<8.8$ & 167 & $2.47(0.85,7.26)$ & ref & $0.63(0.17,2.35)$ & \\
\hline$\geq 8.8$ & 169 & $3.58(1.49,8.64)$ & ref & $0.99(0.33,2.94)$ & \\
\hline RDW, \% & & & & & 0.873 \\
\hline$<14.4$ & 159 & $3.58(1.26,10.17)$ & ref & $0.84(0.23,3.12)$ & \\
\hline$\geq 14.4$ & 177 & $2.65(1.08,6.50)$ & ref & $0.74(0.25,2.22)$ & \\
\hline Hematocrit, \% & & & & & 0.985 \\
\hline$<28.9$ & 167 & $2.91(1.21,7.01)$ & ref & $0.80(0.25,2.5 \mathrm{I})$ & \\
\hline$\geq 28.9$ & 169 & $3.16(1.08,9.26)$ & ref & $0.83(0.24,2.85)$ & \\
\hline $\mathrm{Hb}, \mathrm{g} / \mathrm{dL}$ & & & & & 0.895 \\
\hline$<9.7$ & 163 & $2.75(1.15,6.60)$ & ref & $0.65(0.19,2.23)$ & \\
\hline$\geq 9.7$ & 173 & $3.25(I .09,9.7 I)$ & ref & $0.99(0.30,3.23)$ & \\
\hline $\mathrm{RBC}, 10^{3} / \mu \mathrm{L}$ & & & & & 0.254 \\
\hline$<3.66$ & 167 & $5.46(1.9,16.06)$ & ref & $1.30(0.33,5.21)$ & \\
\hline$\geq 3.66$ & 169 & I.67 $(0.63,4.46)$ & ref & $0.55(0.19,1.58)$ & \\
\hline
\end{tabular}

(Continued) 
Table 3 (Continued).

\begin{tabular}{|c|c|c|c|c|c|}
\hline & \multirow[t]{2}{*}{ No. of Patients } & \multicolumn{3}{|c|}{ Serum $\mathrm{HCO}^{-}$Levels $(\mathrm{mmol} / \mathrm{L})$} & \multirow[t]{2}{*}{ P for Interaction } \\
\hline & & $<22$ & $22-24$ & $>24$ & \\
\hline $\begin{array}{l}\text { Platelet, } 10^{3} / \mu \mathrm{L} \\
\quad<164 \\
\quad \geq 164\end{array}$ & $\begin{array}{l}165 \\
171\end{array}$ & $\begin{array}{c}4.06(1.50,11.00) \\
2.29(0.87,6.03)\end{array}$ & $\begin{array}{l}\text { ref } \\
\text { ref }\end{array}$ & $\begin{array}{l}1.29(0.37,4.45) \\
0.53(0.17,1.67)\end{array}$ & 0.561 \\
\hline $\begin{array}{l}\text { Anion gap, } \mathrm{mmol} / \mathrm{L} \\
\quad<12 \\
\geq 12\end{array}$ & $\begin{array}{l}126 \\
210\end{array}$ & $\begin{array}{l}I .42(0.4 I, 4.9 I) \\
4.18(1.79,9.80)\end{array}$ & $\begin{array}{l}\text { ref } \\
\text { ref }\end{array}$ & $\begin{array}{l}0.63(0.18,2.18) \\
0.89(0.28,2.81)\end{array}$ & 0.313 \\
\hline $\begin{array}{c}\mathrm{pH} \text { value } \\
\quad<7.39 \\
\geq 7.39\end{array}$ & $\begin{array}{l}167 \\
169\end{array}$ & $\begin{array}{c}6.19(2.15,10.74) \\
2.63(1.39,6.18)\end{array}$ & $\begin{array}{l}\text { ref } \\
\text { ref }\end{array}$ & $\begin{array}{l}0.68(0.58,3.20) \\
0.87(0.31,2.01)\end{array}$ & 0.072 \\
\hline $\begin{array}{l}\text { Lactate, } \mathrm{mmol} / \mathrm{L} \\
\quad<4.1 \\
\quad \geq 4.1\end{array}$ & $\begin{array}{l}166 \\
170\end{array}$ & $\begin{array}{l}5.13(1.27,8.84) \\
3.11(1.33,5.26)\end{array}$ & $\begin{array}{l}\text { ref } \\
\text { ref }\end{array}$ & $\begin{array}{l}\text { I.I2 }(0.32,3.84) \\
0.91(0.33,2.50)\end{array}$ & 0.311 \\
\hline $\begin{array}{l}\mathrm{BUN}, \mathrm{mg} / \mathrm{dL} \\
\quad<16 \\
\quad \geq 16\end{array}$ & $\begin{array}{l}153 \\
183\end{array}$ & $\begin{array}{c}3.19(0.80,12.74) \\
2.83(1.29,6.18)\end{array}$ & $\begin{array}{l}\text { ref } \\
\text { ref }\end{array}$ & $\begin{array}{l}0.92(0.19,4.57) \\
0.76(0.28,2.04)\end{array}$ & 0.979 \\
\hline $\begin{array}{l}\text { Creatinine, } \mathrm{mg} / \mathrm{dL} \\
\quad<1 \\
\geq 1\end{array}$ & $\begin{array}{l}132 \\
204\end{array}$ & $\begin{array}{c}4.12(1.24,13.70) \\
2.65(1.16,6.04)\end{array}$ & $\begin{array}{l}\text { ref } \\
\text { ref }\end{array}$ & $\begin{array}{l}1.12(0.32,3.95) \\
0.58(0.17,1.91)\end{array}$ & 0.735 \\
\hline $\begin{array}{l}\text { Chloride, } \mathrm{mmol} / \mathrm{L} \\
\quad<103 \\
\geq 103\end{array}$ & $\begin{array}{l}167 \\
169\end{array}$ & $\begin{array}{c}2.36(0.90,6.19) \\
4.00(1.47,10.84)\end{array}$ & $\begin{array}{l}\text { ref } \\
\text { ref }\end{array}$ & $\begin{array}{l}0.69(0.24,1.97) \\
0.82(0.20,3.45)\end{array}$ & 0.719 \\
\hline $\begin{array}{l}\text { Glucose, } \mathrm{mg} / \mathrm{dL} \\
\quad<103 \\
\geq 103\end{array}$ & $\begin{array}{l}167 \\
169\end{array}$ & $\begin{array}{c}3.29(1.05,10.34) \\
3.10(1.33,7.26)\end{array}$ & $\begin{array}{l}\text { ref } \\
\text { ref }\end{array}$ & $\begin{array}{l}0.65(0.15,2.90) \\
0.91(0.33,2.50)\end{array}$ & 0.876 \\
\hline $\begin{array}{r}\text { APTT, s } \\
<28.1 \\
\geq 28.1\end{array}$ & $\begin{array}{l}167 \\
169\end{array}$ & $\begin{array}{l}2.82(1.11,7.16) \\
3.33(1.21,9.17)\end{array}$ & $\begin{array}{l}\text { ref } \\
\text { ref }\end{array}$ & $\begin{array}{l}1.03(0.35,3.06) \\
0.60(0.16,2.22)\end{array}$ & 0.59 \\
\hline $\begin{array}{l}\text { INR } \\
\qquad 1.2 \\
\geq 1.2\end{array}$ & $\begin{array}{l}152 \\
184\end{array}$ & $\begin{array}{l}3.6 I(1.39,9.4 I) \\
2.69(1.02,7.06)\end{array}$ & $\begin{array}{l}\text { ref } \\
\text { ref }\end{array}$ & $\begin{array}{l}0.63(0.18,2.22) \\
0.96(0.31,2.96)\end{array}$ & 0.628 \\
\hline $\begin{array}{l}\text { PT, s } \\
\quad<13.4 \\
\geq 13.4\end{array}$ & $\begin{array}{l}165 \\
171\end{array}$ & $\begin{array}{l}3.19(1.21,8.40) \\
2.96(1.14,7.69)\end{array}$ & $\begin{array}{l}\text { ref } \\
\text { ref }\end{array}$ & $\begin{array}{l}0.62(0.18,2.20) \\
0.97(0.31,3.01)\end{array}$ & 0.775 \\
\hline $\begin{array}{l}\text { Sodium, } \mathrm{mmol} / \mathrm{L} \\
\quad<137 \\
\geq 137\end{array}$ & $\begin{array}{l}166 \\
170\end{array}$ & $\begin{array}{l}3.22(1.26,8.23) \\
2.92(1.08,7.91)\end{array}$ & $\begin{array}{l}\text { ref } \\
\text { ref }\end{array}$ & $\begin{array}{l}1.03(0.32,3.38) \\
0.63(0.19,2.08)\end{array}$ & 0.831 \\
\hline $\begin{array}{l}\text { Potassium, } \mathrm{mmol} / \mathrm{L} \\
\quad<3.6 \\
\geq 3.6\end{array}$ & $\begin{array}{l}143 \\
193\end{array}$ & $\begin{array}{c}4.40(1.23,15.77) \\
2.65(1.17,6.00)\end{array}$ & $\begin{array}{l}\text { ref } \\
\text { ref }\end{array}$ & $\begin{array}{l}0.8 I(0.16,4.03) \\
0.83(0.3 I, 2.22)\end{array}$ & 0.726 \\
\hline $\begin{array}{l}\text { Total calcium, } \mathrm{mg} / \mathrm{dL} \\
\quad<8.5\end{array}$ & 152 & $6.16(1.75,21.63)$ & ref & $1.46(0.33,6.51)$ & 0.332 \\
\hline
\end{tabular}


Table 3 (Continued).

\begin{tabular}{|c|c|c|c|c|c|}
\hline & \multirow[t]{2}{*}{ No. of Patients } & \multicolumn{3}{|c|}{ Serum $\mathrm{HCO}^{-}$Levels $(\mathrm{mmol} / \mathrm{L})$} & \multirow[t]{2}{*}{$P$ for Interaction } \\
\hline & & $<22$ & $22-24$ & $>24$ & \\
\hline$\geq 8.5$ & 184 & $2.06(0.89,4.756)$ & ref & $0.57(0.20,1.59)$ & \\
\hline $\begin{array}{l}\text { Magnesium, mg/dL } \\
\quad<2 \\
\geq 2\end{array}$ & $\begin{array}{l}119 \\
217\end{array}$ & $\begin{array}{c}2.20(0.82,5.91) \\
4.32(1.60,11.64)\end{array}$ & $\begin{array}{l}\text { ref } \\
\text { ref }\end{array}$ & $\begin{array}{l}0.48(0.12,1.84) \\
1.2 \text { । }(0.38,3.82)\end{array}$ & 0.521 \\
\hline $\begin{array}{l}\text { SBP, } \mathrm{mmHg} \\
\quad<|5| \\
\quad \geq|5|\end{array}$ & $\begin{array}{l}166 \\
170\end{array}$ & $\begin{array}{l}3.27(1.17,9.18) \\
2.96(1.19,7.32)\end{array}$ & $\begin{array}{l}\text { ref } \\
\text { ref }\end{array}$ & $\begin{array}{l}1.09(0.33,3.57) \\
0.56(0.17,1.93)\end{array}$ & 0.728 \\
\hline $\begin{array}{l}\text { DBP, } \mathrm{mmHg} \\
\qquad<81 \\
\quad \geq 81\end{array}$ & $\begin{array}{l}162 \\
174\end{array}$ & $\begin{array}{l}2.92(1.00,8.55) \\
3.10(1.28,7.48)\end{array}$ & $\begin{array}{l}\text { ref } \\
\text { ref }\end{array}$ & $\begin{array}{l}1.13(0.34,3.69) \\
0.55(0.16,1.87)\end{array}$ & 0.561 \\
\hline $\begin{array}{l}\text { MBP, } \mathrm{mmHg} \\
\quad<102 \\
\quad \geq 102\end{array}$ & $\begin{array}{l}162 \\
174\end{array}$ & $\begin{array}{l}2.57(0.79,8.35) \\
3.62(1.57,8.34)\end{array}$ & $\begin{array}{l}\text { ref } \\
\text { ref }\end{array}$ & $\begin{array}{l}1.46(0.43,4.98) \\
0.40(0.11,1.50)\end{array}$ & 0.102 \\
\hline $\begin{array}{l}\text { HR, beats/min } \\
\qquad \begin{array}{l}<0 \\
\geq 90\end{array}\end{array}$ & $\begin{array}{l}162 \\
174\end{array}$ & $\begin{array}{c}3.59(1.12,11.44) \\
2.76(1.19,6.40)\end{array}$ & $\begin{array}{l}\text { ref } \\
\text { ref }\end{array}$ & $\begin{array}{l}0.83(0.21,3.30) \\
0.82(0.29,2.37)\end{array}$ & 0.914 \\
\hline $\begin{array}{l}\text { RR, times/min } \\
\quad<25 \\
\quad \geq 25\end{array}$ & $\begin{array}{l}155 \\
181\end{array}$ & $\begin{array}{c}3.17(0.79,12.68) \\
2.76(1.26,6.03)\end{array}$ & $\begin{array}{l}\text { ref } \\
\text { ref }\end{array}$ & $\begin{array}{l}0.35(0.04,3.40) \\
0.86(0.34,2.15)\end{array}$ & 0.599 \\
\hline $\begin{array}{l}\text { Temperature, }{ }^{\circ} \mathrm{C} \\
\quad<37.28 \\
\geq 37.28\end{array}$ & $\begin{array}{l}166 \\
170\end{array}$ & $\begin{array}{c}2.22(0.97,5.07) \\
5.59(1.58,19.82)\end{array}$ & $\begin{array}{l}\text { ref } \\
\text { ref }\end{array}$ & $\begin{array}{l}0.79(0.30,2.04) \\
0.67(0.11,4.03)\end{array}$ & 0.293 \\
\hline $\begin{array}{c}\mathrm{SPO}_{2}, \% \\
\quad<92 \\
\geq 92\end{array}$ & $\begin{array}{l}135 \\
201\end{array}$ & $\begin{array}{c}2.4 \mathrm{I}(0.98,5.92) \\
3.63(\mathrm{I} .28,10.3 \mathrm{I})\end{array}$ & $\begin{array}{l}\text { ref } \\
\text { ref }\end{array}$ & $\begin{array}{l}0.65(0.22,1.93) \\
0.87(0.23,3.24)\end{array}$ & 0.853 \\
\hline
\end{tabular}

variables PT and hematocrit were eliminated in further analysis. Then Cox regression analysis was conducted. In model 1, after adjusting for age, gender, ethnicity and insurance, compared with the reference group (mid$\mathrm{HCO}^{-}$: $\left.22-24 \mathrm{mmol} / \mathrm{L}\right)$, low $\mathrm{HCO}^{-}$level was a significant predictor of 30-day, 90-day, 1-year and 5-year mortality in AAD patients (HR, 95\% CI: 3.03, 1.53$6.03 ; 3.26,1.74-6.13 ; 2.44,1.40-4.25 ; 1.99,1.24-3.20)$. In model 2, after adjusting for age, gender, ethnicity, insurance, Stanford type, treatment strategy, ICU MV; hypertension; diabetes; hypercholesterolemia; valvular disease; malignancy; stroke; coronary artery disease (CAD); congestive heart failure (CHF); atrial fibrillation; renal disease; liver disease; respiratory disease; congestive heart failure (AKI); white blood cell (WBC); Hb; RBC; PLT; BUN; creatinine; chloride; glucose; activated partial thromboplastin time (APTT); international normalized ratio (INR); sodium; potassium; total $\mathrm{Ca}$; magnesium; anion gap; lactate; $\mathrm{pH}$ value; $\mathrm{SBP}$; DBP; MBP; HR; RR; temperature and $\mathrm{SPO}_{2}$, low $\mathrm{HCO}^{-}$level remained a significant predictor of 30day, 90-day, 1-year and 5-year mortality (HR, 95\% CI: $5.11,1.41-18.46 ; 5.58,1.86-16.77 ; 3.30,1.24-8.78$; $4.08,1.70-9.78)$. We additionally analyzed type $\mathrm{A}$ and B AAD separately, and the results are listed in Supplemental Table 1. The results showed that the HR were significant in both type $\mathrm{A}$ and type $\mathrm{B} \mathrm{AAD}$, and $\mathrm{HR}$ of $\mathrm{HCO}^{-}$in type A AAD group were higher. 
Table 4 Area Under Receiver Operating Characteristic Curve of $\mathrm{HCO}^{-}$and Severity Scores

\begin{tabular}{|l|l|}
\hline Terms & AUC \\
\hline 30-day mortality & 0.69 \\
\hline HCO3 $^{-}$ & 0.68 \\
SAPSII & 0.71 \\
OASIS & 0.58 \\
SOFA & 0.57 \\
SIRS & \\
\hline 90-day mortality & 0.68 \\
\hline HCO3 & \\
SAPSII & 0.70 \\
OASIS & 0.74 \\
SOFA & 0.60 \\
SIRS & 0.55 \\
\hline I-year mortality & \\
\hline HCO3 & \\
SAPSII & 0.68 \\
OASIS & 0.7 I \\
SOFA & 0.72 \\
SIRS & 0.64 \\
\hline 5-year mortality & 0.56 \\
\hline HCO3 & \\
SAPSII & 0.65 \\
OASIS & 0.65 \\
SOFA & 0.58 \\
SIRS & 0.57 \\
\hline NoteP valu & \\
\hline
\end{tabular}

Note: $P$ value $<0.05$.

Abbreviation: AUC, area under curve.

\section{Serum $\mathrm{HCO}^{-}$Is an Effective Predictor of Short-Term and Long-Term Mortality of AAD}

The results from subgroup analysis indicated that most strata had no interaction ( $P$ for interaction $=0.09-0.98$ ). Only patients with liver disease had a higher risk with a low-HCO3- (HR 2.01, 95\% CI 1.24-3.27) (Table 3). In addition, the $\mathrm{AUC}$ of $\mathrm{HCO}^{-}$level in predicting mortality in each period ( 30 days, 90 days, 1 year and 5 years) ranged from 0.60 to 0.68 , which revealed a similar performance to SAPSII, OASIS and a better performance than SOFA and SIRS (Table 4). Moreover, ROC curve analysis and accordingly Kaplan-Meier curves with corresponding $\mathrm{HCO}^{-}$cut-off value were performed respectively (Supplemental Figure 1: A and B: 30-day, C and D: 90day, E and F:1-year and G and H:5-year). Anion gap and lactate were also identified as a biomarkers of the homeostasis of acid-base and had a contribution to the outcome of AAD (Supplemental Table 2). But $\mathrm{HCO}^{-}$had the highest AUC (0.683 versus 0.607 and 0.606 in Supplemental Figure 2).

\section{Discussion}

AAD is a severe cardiovascular disorder characterized by acute onset, rapid progression as well as high rates of complications and mortality. ${ }^{12}$ Especially when AAD patients were transferred into ICU of the hospital, the mortality rate was increased hourly, which makes AAD a challenge in both internal medicine intervention and surgical repair. Currently, simple and effective biomarkers for evaluating risk of complications and mortality of AAD remain lacking. Therefore, the exploration of new indicators is crucial for the death-risk stratification of AAD patients in ICU. In our study, we found that serum $\mathrm{HCO}^{-}$level measured at ICU admission is an independent predictor of short-term and long-term mortality in patients with AAD.

Serum $\mathrm{HCO}^{-}$level reflects the acid-base homeostasis in the human body and is typically included in routine biochemical tests, especially when renal disease progresses. However, some clinical researches indicated a possible role of serum $\mathrm{HCO}^{-}$level, which could be used to predict mortality from diseases other than progressive renal disease. For example, a low $\mathrm{HCO}^{-}$level was associated with malignancy-related mortality, whereas a high $\mathrm{HCO}^{-}$level was related to mortality and complications with cardiovascular diseases. ${ }^{11}$ So far, there has been no research regarding the relationship between serum $\mathrm{HCO}^{-}$level and clinical outcomes of AAD patients. Hence, we evaluated the associations between serum $\mathrm{HCO}^{-}$and all-cause mortality of patients with AAD. Our results indicated that low $\mathrm{HCO}^{-}$levels were related to high all-cause mortality (both short-term and longterm). Moreover, subgroup analysis proved excellent in predicting 30-day, 90-day, 1-year and 5-year mortality, and AUC analysis revealed a similar performance to SAPSII and OASIS and a better performance than SOFA, SIRS score, anion gap and lactate.

We hold that our study is distinct and meaningful, although some previous studies have reported better value ROC compared to $\mathrm{HCO}^{-}$in our study., ${ }^{3,13}$ In these studies, the outcomes were in-hospital mortality and hospital complications such as aortic rupture, mechanical ventilation, renal dysfunction and visceral 
hemorrhage. In contrast, survival time and longer-term mortality (30 days, 90 days, 1 year and 5 years) were set as our outcomes in this study.

Moreover, low serum $\mathrm{HCO}^{-}$is a kind of index of acidosis. It has been proved that acidosis could decrease myocardial contraction and alter systemic vascular resistance promoting to aggravation of circulatory shock, leading to progressive cellular hypoxia and tissue malperfusion, and eventually causing end-organ failure including acute kidney injury. Therefore, it could be the main reason of poor prognosis in critically ill patients of AAD.

Our study is the first to prove the potential value of $\mathrm{HCO}^{-}$levels in predicting short-term and long-term outcomes in AAD patients. We also provided the evidence of the application of a routinely measured serum $\mathrm{HCO}^{-}$as a prognostic indicator, which could improve the adjustment of therapy and potentially reduce mortality rate. Recently, many indicators were proved to be closely associated to the short-term and long-term outcomes of AAD, including Creactive protein (CRP), ${ }^{14}$ metallomatrix protease (MMPs) ${ }^{15}$ and serum amyloid A protein. ${ }^{16}$ Moreover, many risk assessment models to AAD were constructed. ${ }^{17,18}$ The exploration and confirmation of the association between the mortality and serum $\mathrm{HCO}^{-}{ }^{-}$could provide a meaningful candidate of future composite prediction models of mortality of AAD. However, several limitations in our study exist. Firstly, the population size was relatively small due to the limited population source from a single-center database. Moreover, because the serum $\mathrm{HCO}^{-}$levels were shown as integers (as is common laboratory practice), it limited the ability to define precise trisection boundaries. Thirdly, some kinds of data were censored in the MIMIC database, such as the time from onset to admission. Furthermore, we should carry out more in-depth mechanism research in our future work.

\section{Conclusion}

The low admission serum $\mathrm{HCO}^{-}$level is an independent index to predict the short-term and long-term mortality in ICU patients with AAD.

\section{Data Sharing Statement}

Limited access in MIMIC III database.

\section{Funding}

There is no funding to report.

\section{Disclosure}

The authors report no conflicts of interest in this work.

\section{References}

1. Nienaber CA, Clough RE, Sakalihasan N, et al. Aortic dissection. Nat Rev Dis Primers. 2016;2(1):16053. doi:10.1038/nrdp.2016.53

2. Evangelista A, Isselbacher EM, Bossone E, et al. Insights from the international registry of acute aortic dissection: a 20 -year experience of collaborative clinical research. Circulation. 2018;137(17):18461860. doi:10.1161/CIRCULATIONAHA.117.031264

3. Lin Y, Peng Y, Chen Y, et al. Association of lymphocyte to monocyte ratio and risk of in-hospital mortality in patients with acute type A aortic dissection. Biomark Med. 2019;13(15):1263-1272. doi:10.2217/bmm-2018-0423

4. Erbel R. Hypotensive systolic blood pressure predicts severe complications and in-hospital mortality in acute aortic dissection. $J$ Am Coll Cardiol. 2018;71(13):1441-1443. doi:10.1016/j.jacc.2018.02.028

5. Vrsalovic M, Vrsalovic PA, Aboyans V. N-terminal pro-brain natriuretic peptide and short-term mortality in acute aortic dissection: a meta-analysis. Clin Cardiol. 2020;43(11):1255-1259. doi:10.1002/ clc. 23436

6. Vrsalovic M, Vrsalovic PA. Admission C-reactive protein and outcomes in acute aortic dissection: a systematic review. Croat Med J. 2019;60(4):309-315. doi:10.3325/cmj.2019.60.309

7. Vrsalovic M. Prognostic effect of cardiac troponin elevation in acute aortic dissection: a meta-analysis. Int J Cardiol. 2016;214:277-278. doi:10.1016/j.ijcard.2016.03.230

8. Guan X, Gong M, Wang X, et al. Low preoperative fibrinogen level is risk factor for neurological complications in acute aortic dissection. Medicine (Baltimore). 2018;97(21):e10830. doi:10.1097/ MD.0000000000010830

9. Navaneethan SD, Schold JD, Arrigain S, et al. Serum bicarbonate and mortality in stage 3 and stage 4 chronic kidney disease. Clin $\mathrm{J} \mathrm{Am}$ Soc Nephrol. 2011;6(10):2395-2402. doi:10.2215/CJN.03730411

10. Schutte E, Lambers HH, Lutgers HL, et al. Serum bicarbonate and kidney disease progression and cardiovascular outcome in patients with diabetic nephropathy: a post hoc analysis of the RENAAL (Reduction of End Points in Non-Insulin-Dependent Diabetes With the Angiotensin II Antagonist Losartan) Study and IDNT (Irbesartan Diabetic Nephropathy Trial). Am J Kidney Dis. 2015;66:450-458.

11. Al-Kindi SG, Sarode A, Zullo M, et al. Serum bicarbonate concentration and cause-specific mortality: the National Health and Nutrition Examination Survey 1999-2010. Mayo Clin Proc. 2020;95(1):113-123. doi:10.1016/j.mayocp.2019.05.036

12. Johnson AE, Pollard TJ, Shen L, et al. MIMIC-III, a freely accessible critical care database. Sci Data. 2016;3:160035. doi:10.1038/ sdata.2016.35

13. Yang G, Zhou Y, He H, Pan X, Chai X. Ischemia-modified albumin, a novel predictive marker of in-hospital mortality in acute aortic dissection patients. Front Physiol. 2019;10:1253. doi:10.3389/ fphys.2019.01253

14. Wen D, Du X, Dong JZ, Zhou XL, Ma CS. Value of D-dimer and $\mathrm{C}$ reactive protein in predicting inhospital death in acute aortic dissection. Heart. 2013;99:1192-1197. doi:10.1136/heartjnl-2013304158

15. Proietta M, Tritapepe L, Cifani N, Ferri L, Taurino M, Del PF. MMP12 as a new marker of Stanford-A acute aortic dissection. Ann Med. 2014;46:44-48. doi:10.3109/07853890.2013.876728

16. He Y, Ma C, Xing J, et al. Serum amyloid a protein as a potential biomarker in predicting acute onset and association with in-hospital death in acute aortic dissection. BMC Cardiovasc Disord. 2019;19:282. doi:10.1186/s12872-019-1267-0 
17. Yang G, Zhou Y, He H, Pan X, Li X, Chai X. A nomogram for predicting in-hospital mortality in acute type A aortic dissection patients. $J$ Thorac Dis. 2020;12:264-275. doi:10.21037/ jtd.2020.01.41
18. Ong CS, Nam L, Yesantharao P, et al. The strongest risk factor for operative mortality in acute type A aortic dissection is acidosis: validation of risk model. Semin Thorac Cardiovasc Surg. 2020;32 (4):674-680. doi:10.1053/j.semtcvs.2020.02.023

\section{Publish your work in this journal}

The International Journal of General Medicine is an international, peer-reviewed open-access journal that focuses on general and internal medicine, pathogenesis, epidemiology, diagnosis, monitoring and treatment protocols. The journal is characterized by the rapid reporting of reviews, original research and clinical studies across all disease areas. The manuscript management system is completely online and includes a very quick and fair peer-review system, which is all easy to use. Visit http://www.dovepress.com/ testimonials.php to read real quotes from published authors.

Submit your manuscript here: https://www.dovepress.com/international-journal-of-general-medicine-journal 